hep-ph/9808254

TIT-HEP-397

KEK-TH-582

\title{
The Singular Seesaw Mechanism with Hierarchical Dirac Neutrino Mass
}

\author{
Yuichi Chikira* \\ Department of Physics, Tokyo Institute of Technology \\ Oh-okayama, Meguro, Tokyo 152-0033, Japan \\ Naoyuki Haba ${ }^{\dagger}$ \\ Faculty of Engineering, Mie University, \\ Mie, 514-0008, Japan \\ Yukihiro Mimura ${ }^{\ddagger}$ \\ Theory Group, KEK, Oho 1-1, Tsukuba, Ibaraki 305-0801, Japan
}

(September 16, 2018)

\begin{abstract}
The singular seesaw mechanism can naturally explain the atmospheric neutrino deficit by the maximal oscillation between $\nu_{\mu_{L}}$ and $\nu_{\mu_{R}}$. This mechanism can also induce three different scales of neutrino mass squared differences, which can explain the neutrino deficits of three independent experiments (solar, atmospheric, and LSND) by neutrino oscillations. In this paper we show that the realistic mixing angles among neutrinos can be obtained by introducing the hierarchy in the Dirac neutrino mass. In the case where Majorana neutrino mass matrix has rank 2, the solar neutrino deficit is explained by the vacuum oscillation between $\nu_{e}$ and $\nu_{\tau}$. We also consider the case where Majorana neutrino mass matrix has rank 1. In this case, the mater enhanced Mikheyev-Smirnov-Wolfenstein solar neutrino solution is prefered as the solution of the solar neutrino deficit.
\end{abstract} 4.60.Pq, 14.60.St

\footnotetext{
*e-mail: ychikira@th.phys.titech.ac.jp

†e-mail: haba@phen.mie-u.ac.jp

$\ddagger$ e-mail: mimura@theory.kek.jp
} 


\section{INTRODUCTION}

According to the recent Super-Kamiokande experiment [1], their atmospheric neutrino data indicate the oscillation between $\nu_{\mu}$ and $\nu_{\tau}$ or sterile neutrinos with the maximal mixing

$$
\sin ^{2} 2 \theta_{\mu x} \sim 1
$$

where $x$ represents $\tau$ or sterile neutrinos. The neutrino mass squared difference $\Delta m_{\mathrm{atm}}^{2}$ is of order $10^{-3}\left[\mathrm{eV}^{2}\right]$.

It is well-known that other two independent experiments also imply neutrino oscillations. One is the solar neutrino experiment. This experiment implies the oscillation between $\nu_{e}$ and other neutrinos, and there are three possible solutions, namely, large or small mixing angle Mikheyev-Smirnov-Wolfenstein (MSW) solution [2], and the vacuum oscillation solution [3]. The small (large) angle MSW solution suggests [4]

$$
\sin ^{2} 2 \theta_{e x} \sim 10^{-2}(0.4-1)
$$

with mass squared difference of order $10^{-5}\left[\mathrm{eV}^{2}\right]$, and the vacuum oscillation solution suggests

$$
\sin ^{2} 2 \theta_{\text {ex }} \sim 0.75-1
$$

with the mass squared difference of order $10^{-10}\left[\mathrm{eV}^{2}\right]$. The vacuum oscillation solution is now the most suitable solution from the electron energy spectrum of the recent SuperKamiokande experimental data [5], although the small angle MSW solution has been regarded as the most realistic candidate. On the other hand, LSND experiment measures oscillation between $\bar{\nu}_{\mu}$ and $\bar{\nu}_{e}$ [6] with a short base line experiment. Although the confirmation of the LSND results still awaits future experiments, $\mathbb{\text { f }}$ their results indicate the small mixing angle

$$
\sin ^{2} 2 \theta_{\mu e} \sim 10^{-2}
$$

with mass squared difference $\Delta m_{\mathrm{LSND}}^{2} \sim 1\left[\mathrm{eV}^{2}\right]$.

The most interesting mechanism which can naturally explain the smallness of neutrino masses is the so-called seesaw mechanism [8]. The general mass matrix of neutrinos above $S U(2)_{L}$ breaking is given by

$$
\mathcal{M}=\left(\begin{array}{cc}
0 & m_{D} \\
m_{D}^{T} & M_{R}
\end{array}\right),
$$

where $m_{D}$ and $M_{R}$ represent Dirac and Majorana $3 \times 3$ flavor space mass matrices, respectively. In the case of $m_{D} \ll M_{R}$, there appear three light neutrinos with mass matrix

$$
\mathcal{M}_{\text {light }}=-m_{D} M_{R}^{-1} m_{D}^{T} .
$$

This is the essence of the seesaw mechanism. It is worth noting that here it is assumed that there exists inverse matrix of $M_{R}^{-1}$, that is, $\operatorname{det} M_{R} \neq 0$. The singular seesaw mechanism

${ }^{1}$ Recent measurements in the KARMEN detector exclude part of the LSND allowed region [7]. 
[9,10], which is also called "partially broken seesaw mechanism" [11] [ is just the case of $\operatorname{det} M_{R}=0$. Then, some light right-handed neutrinos are not integrated out, and behave as sterile neutrinos. It turns out that mixings between the survived sterile neutrinos and active neutrinos are large in general because of the pseudo-Dirac texture [12]. We can use this mechanism to explain the large mixing of the atmospheric neutrino experiment. If nature adopts four (or more) neutrino oscillations, the singular seesaw mechanism supplies one of the most attractive models.

The authors of Ref. [9] discussed this singular seesaw mechanism in the case that there is no hierarchy in the Dirac mass matrix $m_{D}$ and Majorana mass matrix $M_{R}$. They did not take the small mixing of the LSND into account. In this paper, we study the singular seesaw mechanism by introducing the hierarchy in the Dirac mass matrix $m_{D}$ in order to explain the small mixing of the LSND experiment. We will also study whether the hierarchical Dirac mass can induce not only the small mixing of the LSND experiment but also the small mixing of the MSW solar neutrino solution.

This paper is organized as follows: In section II, we will review the singular seesaw mechanism briefly. In section III, we introduce hierarchical Dirac mass matrix, and determine the order of parameters. We show that the vacuum oscillation solution is prefered as the solution of the solar neutrino deficit in the case where Majorana neutrino mass matrix has rank 2 and that the MSW solution is prefered in the case where Majorana neutrino mass matrix has rank 1 . In section IV, we give summary and discussions.

\section{SINGULAR SEESAW MECHANISM}

At first, we explain the pseudo-Dirac mass texture [12]. In the one generation the neutrino mass term above $S U(2)_{L}$ breaking is given by

$$
-\mathcal{L}=\frac{1}{2}\left(\begin{array}{ll}
\nu & \nu^{C}
\end{array}\right)\left(\begin{array}{cc}
0 & m \\
m & M
\end{array}\right)\left(\begin{array}{c}
\nu \\
\nu^{C}
\end{array}\right)
$$

where $\nu$ and $\nu^{C}$ represent (two component) left- and right-handed neutrinos, respectively. Here we consider the case of $M \ll m$. In this case the mass matrix (7) realizes large mixing angle of $\sin ^{2} 2 \theta=\frac{m^{2}}{m^{2}+M^{2} / 4} \sim 1$ between $\nu$ and $\nu^{C}$. The eigenvalues of this mass matrix are $\pm m+M / 2$, and the neutrino mass squared difference is $\Delta m^{2}=2 m M$ [12]. This mass term is almost Dirac but not exact, so it is called pseudo-Dirac texture, which can naturally induce the maximal mixing. The mass term in the opposite case of $M \gg m$ is that of ordinary seesaw mechanism.

Now let us take three generations into consideration. We take $m$ and $M$ as $3 \times 3$ matrices $m_{D}$ and $M_{M}$, respectively in Eq.(17). The right-handed Majorana neutrino mass matrix $M_{M}$ is assumed to be rank 2 (or 1). In this case two (one) neutrinos become light by the ordinary seesaw mechanism, and remaining one (two) neutrino has the pseudo-Dirac mass texture. For example, in the rank-2 case, we can obtain the eigenvalues of four light neutrinos [9, 10] as

\footnotetext{
${ }^{2}$ In this paper we call this mechanism "the singular seesaw mechanism".
} 


$$
\beta m, \quad \beta m, \quad \text { and } \pm m+\beta m,
$$

where $\beta=m / M$, in the case of no hierarchy in the mass matrices $m_{D}$ and $M_{M}$. It is interesting that the two lighter neutrinos' masses and the mass splitting for the pseudoDirac neutrinos are the same scale. Then three mass squared differences form geometric series as

$$
\Delta m^{2}=\beta^{2} m^{2}, \quad \beta m^{2}, \quad \text { and } m^{2},
$$

and are favorable to explain three known neutrino oscillation modes, namely, solar neutrinos(MSW solution), atmospheric neutrinos and LSND [10]. Furthermore, since the middle scale of the mass squared difference for atmospheric neutrinos corresponds to pseudo-Dirac neutrinos, its maximal mixing realizes naturally.

However, they can not explain neither the small mixing angle of LSND nor the small angle MSW solution if there is no mass hierarchy in $m_{D}$ and $M_{M}$. We will see that the hierarchical Dirac mass matrices lead to different series of mass squared difference, which is suitable for vacuum oscillation solution for solar neutrino deficit rather than MSW solutions.

The flaws of the singular seesaw mechanism is that the Dirac neutrino mass $m$ needs to be too small (about $1[\mathrm{eV}]$ ), and it fails the motivation of original seesaw mechanism. When we incorporate the singular seesaw mechanism into phenomenological models, we need some extra mechanism to apply the small Dirac neutrino mass. Its smallness will be realized, for example, by the non-renormalizable interactions [13], though we do not mention the detail in this paper.

\section{SINGULAR SEESAW MECHANISM WITH HIERARCHICAL DIRAC NEUTRINO MASS MATRIX}

We introduce the hierarchy in the Dirac neutrino mass matrix as

$$
m_{D}=\left(\begin{array}{ccc}
\epsilon^{\prime} m_{11} & \epsilon^{\prime} m_{12} & \epsilon^{\prime} m_{13} \\
\epsilon m_{21} & \epsilon m_{22} & \epsilon m_{23} \\
m_{31} & m_{32} & m_{33}
\end{array}\right) .
$$

We can take the hierarchical parameter $\epsilon$ and $\epsilon^{\prime}$ as $\epsilon^{\prime} \leq \epsilon<1$, when we do not order the left-handed indices with naming of neutrino flavors. In this paper, we do not mention the hierarchical structure with respect to right-handed indices. The mass term of neutrinos is given by

$$
-\mathcal{L}=m_{D i j} \nu_{i} \nu_{j}^{C}+\frac{1}{2} M_{M i j} \nu_{i}^{C} \nu_{j}^{C}
$$

We analyze this model in two cases, where the rank of Majorana mass matrix $M_{M}$ is 1 or 2 .

At first, we study the case where Majorana mass $M_{M}$ has rank 2 as $M_{M}=$ $\operatorname{diag}\left(M_{1}, M_{2}, 0\right)$. After integrating out the heavy neutrinos ${ }^{n}$, light neutrinos have masses as

\footnotetext{
${ }^{3}$ This heavy neutrino mass $M$ turns out to be $1[\mathrm{keV}]-1[\mathrm{MeV}]$. We integrate out the heavy neutrinos
} 


$$
-\mathcal{L}=-\frac{1}{2}\left(\frac{m_{D i 1} m_{D j 1}}{M_{1}}+\frac{m_{D i 2} m_{D j 2}}{M_{2}}\right) \nu_{i} \nu_{j}+m_{D i 3} \nu_{i} \nu_{3}^{C}
$$

The mass matrix for $\left(\nu_{1}, \nu_{2}, \nu_{3}, \nu_{3}^{C}\right) \equiv(\alpha, \beta, \gamma, s)$ is given by

$$
\mathcal{M} \sim\left(\begin{array}{cccc}
-\epsilon^{\prime 2} \beta & -\epsilon \epsilon^{\prime} \beta & -\epsilon^{\prime} \beta & \epsilon^{\prime} \\
-\epsilon \epsilon^{\prime} \beta & -\epsilon^{2} \beta & -\epsilon \beta & \epsilon \\
-\epsilon^{\prime} \beta & -\epsilon \beta & -\beta & 1 \\
\epsilon^{\prime} & \epsilon & 1 & 0
\end{array}\right) m .
$$

This matrix is diagonalized as

$$
U^{\dagger} \mathcal{M} U \sim \operatorname{diag}\left(\epsilon^{\prime 2} \beta m, \epsilon^{2} \beta m,(1-\beta) m,-(1+\beta) m\right),
$$

where

$$
U \sim\left(\begin{array}{cccc}
1 & -\epsilon^{\prime} / \epsilon & \epsilon^{\prime} & -\epsilon^{\prime} \\
\epsilon^{\prime} / \epsilon & 1 & \epsilon & -\epsilon \\
-\epsilon^{\prime} & -\epsilon & 1 & -1 \\
\epsilon^{\prime} \beta & -\epsilon \beta & 1 & 1
\end{array}\right)
$$

Now we estimate the probability of the neutrino oscillation, which is given by

$$
P\left(\nu_{\alpha} \rightarrow \nu_{\beta}\right)=\delta_{\alpha \beta}-4 \sum_{i<j} U_{\alpha i} U_{\beta i}^{*} U_{\alpha j}^{*} U_{\beta j} \sin ^{2} \frac{\Delta m_{i j}^{2}}{4 E} L,
$$

where we neglect $C P$ phase for simplicity. The oscillation amplitude between $\alpha$ and $\beta$ is given by

$$
-4 \sum_{i<j} U_{\alpha i} U_{\beta i}^{*} U_{\alpha j}^{*} U_{\beta j}
$$

From Eq.(14), we can obtain three scales of mass squared differences of $\Delta m_{12}^{2} \sim \epsilon^{4} \beta^{2} m^{2}$, $\Delta m_{34}^{2} \sim \beta m^{2}$, and $\Delta m_{13}^{2} \sim \Delta m_{14}^{2} \sim \Delta m_{23}^{2} \sim \Delta m_{24}^{2} \sim m^{2}$. We list the amplitudes corresponding to these three oscillation in Table I. The oscillation between $\gamma \leftrightarrow s$ gives a large mixing, which is expected to correspond to atmospheric neutrino oscillation. Then, we fix

$$
\beta m^{2} \sim 10^{-3}\left[\mathrm{eV}^{2}\right]
$$

The oscillation with $\Delta m^{2} \sim m^{2}$ may correspond to LSND data, so we fix

$$
m^{2} \sim 1\left[\mathrm{eV}^{2}\right] .
$$

Then there remain two patterns whether $(\alpha, \beta)$ is assigned as $(e, \tau)$ or $(\tau, e)$. Let us consider both possibilities here.

here though the scale is lower than the momentum scales of neutrino experiments (e.g., about $1[\mathrm{GeV}]$ for atmospheric neutrinos). This integrating out method is used simply because we display our results clearly. Since the heavy neutrinos have very small mixing with light neutrinos, there is considerable validity to our results below. 
(1-1) In the case of $(\alpha, \beta)=(e, \tau), \epsilon^{\prime}$ must be of order $10^{-1}$ from the small mixing of LSND data. Then the oscillation with $\Delta m^{2} \sim \epsilon^{4} \beta^{2} m^{2} \sim 10^{-6} \epsilon^{4}\left[\mathrm{eV}^{2}\right]$ should correspond to the solar neutrino oscillation. (i): For the mass squared difference of MSW solution, we must choose the parameter $\epsilon$ to be close to 1 . Then, it turns out that the $\nu_{\mu}-\nu_{\tau}$ mixing is large with $\Delta m^{2} \sim 1\left[\mathrm{eV}^{2}\right]$. This oscillation leads to contradiction with the atmospheric neutrino data. Therefore we can not obtain the MSW solution in this pattern. (ii): On the other hand, the vacuum oscillation solution can be realized when $\epsilon=O\left(10^{-1}\right)$. We can realize the large mixing of Eq.(3) because the corresponding mixing angle is of order $\left(\epsilon^{\prime} / \epsilon\right)^{2}$.

(1-2) In the case of $(\alpha, \beta)=(\tau, e), \epsilon$ must be of order $10^{-1}$ from the small mixing of LSND data. In this pattern, the mass squared difference corresponding to the solar neutrino oscillation should be of order $10^{-10}\left[\mathrm{eV}^{2}\right]$. Therefore, only vacuum oscillation solution can be allowed. To explain the large mixing of the solution, $\epsilon^{\prime}$ must be satisfy $\epsilon \sim 10^{-1}$. This is the same parameters as the case of $(i i)$ in (1-1).

Next, let us consider the case where Majorana mass $M_{M}$ has rank 1 as $M_{M}=$ $\operatorname{diag}(M, 0,0)$. The mass term of neutrinos is given by

$$
-\mathcal{L}=m_{D i j} \nu_{i} \nu_{j}^{C}+\frac{1}{2} M \nu_{1}^{C} \nu_{1}^{C} .
$$

After integrating $\nu_{1}^{C}$ out, light neutrinos $\left(\nu_{1}, \nu_{2}, \nu_{3}, \nu_{2}^{C}, \nu_{3}^{C}\right) \equiv\left(\alpha, \beta, \gamma, s_{1}, s_{2}\right)$ have masses as

$$
\mathcal{M} \sim\left(\begin{array}{ccccc}
-\epsilon^{\prime 2} \beta & -\epsilon^{\prime} \epsilon \beta & -\epsilon^{\prime} \beta & \epsilon^{\prime} & \epsilon^{\prime} \\
-\epsilon \epsilon^{\prime} \beta & -\epsilon^{2} \beta & -\epsilon \beta & \epsilon & \epsilon \\
-\epsilon^{\prime} \beta & -\epsilon \beta & -\beta & 1 & 1 \\
\epsilon^{\prime} & \epsilon & 1 & 0 & 0 \\
\epsilon^{\prime} & \epsilon & 1 & 0 & 0
\end{array}\right) m .
$$

This matrix can be diagonalized as

$$
U^{\dagger} \mathcal{M} U \sim \operatorname{diag}\left(\epsilon^{\prime 2} \beta m,\left(\epsilon-\epsilon^{2} \beta\right) m,-\left(\epsilon+\epsilon^{2} \beta\right) m,(1-\beta) m,-(1+\beta) m\right),
$$

where

$$
U \sim\left(\begin{array}{ccccc}
1 & -\epsilon^{\prime} / \epsilon & -\epsilon^{\prime} / \epsilon & \epsilon^{\prime} & \epsilon^{\prime} \\
\epsilon^{\prime} / \epsilon & 1 & 1 & \epsilon & \epsilon \\
\epsilon^{\prime} & -\epsilon & -\epsilon & 1 & 1 \\
\epsilon^{\prime} \beta & -1 & 1 & 1 & -1 \\
\epsilon^{\prime} \beta & 1 & -1 & 1 & -1
\end{array}\right) .
$$

There are four scales of mass squared differences as $\Delta m^{2} \sim \epsilon^{3} \beta m^{2}, \beta m^{2}, \epsilon^{2} m^{2}$ and $m^{2}$. The oscillation amplitudes corresponding to these oscillation modes are listed in Table II. The atmospheric neutrino oscillation can be regarded as $\gamma \leftrightarrow s_{1}, s_{2}$. Therefore, the mass squared difference $\beta m^{2}$ must be of order $10^{-3}\left[\mathrm{eV}^{2}\right]$. Then, the mass squared difference corresponding to the solar neutrino oscillation should be $\epsilon^{3} \beta m^{2}\left[\mathrm{eV}^{2}\right] \sim 10^{-3} \epsilon^{3}\left[\mathrm{eV}^{2}\right]$. There are two candidates for the mass squared differences of LSND, namely, $\Delta m_{\mathrm{LSND}}^{2} \sim \epsilon^{2} m^{2}$ or $\Delta m_{\mathrm{LSND}}^{2} \sim m^{2}$. Here we consider the both possibilities. 
(2-1) In the case of $(\alpha, \beta)=(e, \tau), \epsilon^{\prime}$ must be of order $10^{-1}$ from the small mixing of LSND data. As for the solar neutrinos, the vacuum oscillation solution is excluded because the parameter $\epsilon$ can not be smaller than $\epsilon^{\prime} \sim 10^{-1}$. Then we consider the parameter $\epsilon=O\left(10^{-1}\right)$ in order to obtain the suitable mass squared difference for the MSW solution. In this case $\epsilon^{\prime} / \epsilon$ tend to becomes close to 1 , and in this case, the $\nu_{e} \leftrightarrow \nu_{\tau}$ oscillation with $\Delta m^{2} \sim \epsilon^{2} m^{2}$ becomes large mixing. However, the large mixing of the order of $\left(\epsilon^{\prime} / \epsilon\right)^{2} \sim 1$ with $\Delta m^{2}>10^{-3}\left[\mathrm{eV}^{2}\right]$ is excluded from CHOOZ experiment [14], and we should choose the mixing $\left(\epsilon^{\prime} / \epsilon\right)^{2}$ to be smaller than $O\left(10^{-1}\right)$. This choice of parameters leads the solar neutrino solution to the small angle MSW solution. Therefore, $\left(\epsilon^{\prime} / \epsilon\right)^{2}$ appears to be of order $10^{-2}$. In order to get such a $\left(\epsilon^{\prime} / \epsilon\right)^{2}$, we need delicate tuning of parameters.

(2-2) In the case of $(\alpha, \beta)=(\tau, e), \epsilon^{\prime}(\epsilon)$ must be of order $10^{-1}$ from small mixing amplitude of LSND with $\Delta m_{\mathrm{LSND}}^{2} \sim \epsilon^{2} m^{2}\left(\Delta m_{\mathrm{LSND}}^{2} \sim m^{2}\right)$. For the same reason of (2-1), the vacuum oscillation solution is excluded, and the parameter $\epsilon$ should be chosen to be of order $10^{-1}$ for the MSW solution. Though the large angle MSW solution through $\nu_{e} \leftrightarrow \nu_{s}$ oscillation mode seems to be possible, it is not allowed at the 99\% C.L. [15] in two flavor analysis. Therefore, in this case, we can not help but consider other oscillation mode, namely, $\nu_{e} \leftrightarrow \nu_{\tau}$, as the solution for solar neutrino deficit. As we mentioned in (2-1), the small angle MSW solution through $\nu_{e} \leftrightarrow \nu_{\tau}$ oscillation mode seems to be possible. However, since the mixing of $\nu_{e}$ and $\nu_{s}$ is large, we need the detail analysis of three generation mixing in this case.

\section{CONCLUSION}

The recent atmospheric neutrino data of Super-Kamiokande suggests the maximal mixing between $\nu_{\mu}$ and other neutrinos. The singular seesaw mechanism is one of the most interesting scenario that can naturally explain this large mixing angle between $\nu_{\mu_{L}}$ and $\nu_{\mu_{R}}$. This mechanism can also induce three independent mass squared differences, which are suitable for the solutions of the solar and atmospheric neutrino anomalies, and the LSND data. The original scenario in Ref. [9] can not explain neither the small mixing angle of the LSND data nor small angle solution of MSW. Thus, we introduce the hierarchy in the Dirac neutrino mass matrix, and reanalyzed the singular seesaw mechanism. As the results, we can obtain the small mixing solutions of the LSND and MSW as follows.

In the case of rank-2 Majorana mass, the Dirac mass matrix should be the form of

$$
\left(\begin{array}{ccc}
\epsilon m_{e e} & \epsilon m_{e \mu} & \epsilon m_{e \tau} \\
m_{\mu e} & m_{\mu \mu} & m_{\mu \tau} \\
\epsilon m_{\tau e} & \epsilon m_{\tau \mu} & \epsilon m_{\tau \tau}
\end{array}\right),
$$

where dimensionless parameter $\epsilon$ is of order $10^{-1}$ and $m_{\alpha \beta} \sim 1[\mathrm{eV}]$. The non-zero elements of Majorana mass should be of order $1[\mathrm{keV}]$. It is important that the solar neutrino deficit can be explained by the vacuum oscillation between $\nu_{e}$ and $\nu_{\tau}$, in contrast to the original framework of Ref. [9].

In the case of rank-1 Majorana mass, the small angle MSW solution is suitable for the solar neutrino oscillation. The Dirac mass matrix should be 


$$
\left(\begin{array}{ccc}
\epsilon m_{e e} & \epsilon m_{e \mu} & \epsilon m_{e \tau} \\
m_{\mu e} & m_{\mu \mu} & m_{\mu \tau} \\
\epsilon^{\prime} m_{\tau e} & \epsilon^{\prime} m_{\tau \mu} & \epsilon^{\prime} m_{\tau \tau}
\end{array}\right)
$$

where $\epsilon$ is of order $10^{-1}$ and $\epsilon^{\prime}$ should satisfy the condition $\left(\epsilon^{\prime} / \epsilon\right)^{2}<10^{-1}$. There is an extra oscillation mode $\Delta m^{2} \sim 10^{-2}\left[\mathrm{eV}^{2}\right]$ or $\Delta m^{2} \sim 10^{2}\left[\mathrm{eV}^{2}\right]$.

Finally, we would like to comment about Big Bang nucleosynthesis (BBN) in the sterile scenario. The ratio of deuterium to hydrogen and the abundance of ${ }^{4} \mathrm{He}$ are determined by the ratio of neutrons to protons at the time when the weak interaction freeze out. The effective number of light neutrino flavors $N_{\nu}$ contribute to the energy density, which influences the expansion rate. Thus, we can obtain the upper limit of $N_{\nu}$ from the BBN constraint [16]. Although the standard BBN scenario shows $N_{\nu} \leq 3.6$ [16], the large lepton number asymmetry in the early universe may allow $N_{\nu}=4$ [17], which corresponds to the rank- 2 case in this paper. On the other hand, rank-1 case induces $N_{\nu}=5$, since $\nu_{\tau}-\nu_{s}$ mixing is large. Thus this case needs extra mechanism which suppress the effective number of neutrinos in the early universet?

\section{ACKNOWLEDGMENTS}

N.H would like to thank Professor S. Raby and Dr. K. Tobe for helpful discussions. Y.M. wishes to thank Institute for Cosmic Ray Research, University of Tokyo, in which most of this research was carried out. This work was partially supported by JSPS Research Fellowships for Young Scientists (Y.C. and Y.M.).

${ }^{4}$ One possibility is to consider the large uncertainty of the systematic error in ${ }^{4} \mathrm{He}$ abundance 18 . 


\section{REFERENCES}

[1] Super-Kamiokande Collaboration, Y. Fukuda et al., hep-ex/9803006; hep-ex/9805006; hep-ex/9807003; talk by T. Kajita at Neutrino-98, Takayama, Japan, June 1998.

[2] L. Wolfenstein, Phys. Rev. D17, 2369 (1978).

S. P. Mikheyev and A. Yu. Smirnov, Yad. Fiz. 42, 1441 (1985) [Sov. J. Nucl. Phys. 42, 913 (1985)]; Nuovo Cim. 9C, 17 (1986).

[3] See example, V. Barger, R. J. N. Phillips, and K. Whisnant, Phys. Rev. Lett. 69, 3135 (1992).

[4] GALLEX Collaboration, Phys. Lett. B285, 390 (1992).

P. I. Krastev and S. T. Petcov, Phys. Lett. B299, 99 (1993).

G. Fiorentini et. al., Phys. Rev. D49, 6298 (1994).

N. Hata and P. G. Langacker, Phys. Rev. D56, 6107 (1997).

[5] Talk by Y. Suzuki at Neutrino-98, Takayama, Japan, June 1998.

[6] LSND Collaboration, C. Athanassopoulos et al., Phys. Rev. Lett. 75, 2650 (1995); ibid. 77, 3082 (1996); nucl-ex/9706006.

[7] The LSND results will be tested by the KARMEN experiment, talk by B. Armbruster at 33rd Rencontres de Moriond : Electroweak Interactions and Unified Theories, Les Arcs, France, March 1998, and talk by B. Zeitnitz at Neutrino 98, Takayama, Japan, June 1998, and also by the BooNE experiment, E. Church et al., nucl-ex/9706011.

[8] T. Yanagida, in: O. Sawada and A. Sugamoto(eds.), Proc. of the Workshop on the Unified Theory and Baryon Number in the Universe (KEK report 79-18, 1979) p.95.

M. Gell-Mann, P. Ramond, and R. Slansky, in: P. van Nieuwenhuizen and D. Z. Freedman(eds.), Supergravity (North Holland, Amsterdam, 1979) p.315.

[9] E. J. Chun, C. W. Kim, and U. W. Lee, Phys. Rev. D58, 093003 (1998).

[10] S. L. Glashow, Phys. Lett. B256, 255 (1991).

[11] M. Fukugita and T. Yanagida, Phys. Rev. Lett. 66, 2705 (1991).

[12] K. Kobayashi, C. S. Lim and M. M. Nojiri, Phys. Rev. Lett. 67, 1685 (1991).

H. Minakata and H. Nunokawa, Phys. Rev. D45, 3316 (1992).

[13] P. Langacker, Phys. Rev. D58, 093017 (1998).

[14] CHOOZ Collaboration, M. Apollonio et al., Phys. Lett. B420, 397 (1998).

[15] J. N. Bahcall, P. I. Krastev, and A. Yu. Smirnov, hep-ph/9807216.

[16] See for example, N. Hata, G. Steigman, S. Bludman, and P. Langacker, Phys. Rev. D55, 540 (1997).

[17] See for example, R. Foot and R. R. Volkas, Phys. Rev. D56, 6653 (1997).

[18] A. Geiser, hep-ph/9810493. 


\section{TABLES}

\begin{tabular}{lccc}
\hline \hline & $\Delta m^{2} \sim \epsilon^{4} \beta^{2} m^{2}$ & $\Delta m^{2} \sim \beta m^{2}$ & $\Delta m^{2} \sim m^{2}$ \\
\hline$\alpha \leftrightarrow \beta$ & $\left(\frac{\epsilon^{\prime}}{\epsilon}\right)^{2}$ & $\epsilon^{2} \epsilon^{\prime 2}$ & $\epsilon^{\prime 2}$ \\
$\alpha \leftrightarrow \gamma$ & $\epsilon^{\prime 2}$ & $\epsilon^{\prime 2}$ & $\epsilon^{\prime 2}$ \\
$\alpha \leftrightarrow s$ & $\epsilon^{\prime 2} \beta^{2}$ & $\epsilon^{\prime 2}$ & $\epsilon^{\prime 2} \beta$ \\
$\beta \leftrightarrow \gamma$ & $\epsilon^{\prime 2}$ & $\epsilon^{2}$ & $\epsilon^{2}$ \\
$\beta \leftrightarrow s$ & $\epsilon^{\prime 2} \beta^{2}$ & $\epsilon^{2}$ & $\epsilon^{2} \beta$ \\
$\gamma \leftrightarrow s$ & $\epsilon^{\prime 2} \epsilon^{2} \beta^{2}$ & 1 & $\epsilon^{2} \beta$ \\
\hline \hline
\end{tabular}

TABLE I. Oscillation amplitudes in the case of rank-2

\begin{tabular}{ccccc}
\hline \hline & $\Delta m^{2} \sim \epsilon^{3} \beta m^{2}$ & $\Delta m^{2} \sim \beta m^{2}$ & $\Delta m^{2} \sim \epsilon^{2} m^{2}$ & $\Delta m^{2} \sim m^{2}$ \\
\hline$\alpha \leftrightarrow \beta$ & $\left(\frac{\epsilon^{\prime}}{\epsilon}\right)^{2}$ & $\epsilon^{2} \epsilon^{\prime 2}$ & $\left(\frac{\epsilon^{\prime}}{\epsilon}\right)^{2}$ & $\epsilon^{\prime 2}$ \\
$\alpha \leftrightarrow \gamma$ & $\epsilon^{\prime 2}$ & $\epsilon^{\prime 2}$ & $\epsilon^{\prime 2}$ & $\epsilon^{\prime 2}$ \\
$\alpha \leftrightarrow s_{1}, s_{2}$ & $\left(\frac{\epsilon^{\prime}}{\epsilon}\right)^{2}$ & $\epsilon^{\prime 2}$ & $\frac{\epsilon^{\prime 2}}{\epsilon} \beta$ & $\frac{\epsilon^{\prime 2}}{\epsilon}$ \\
$\beta \leftrightarrow \gamma$ & $\epsilon^{2}$ & $\epsilon^{2}$ & $\epsilon^{\prime 2}$ & $\epsilon^{2}$ \\
$\beta \leftrightarrow s_{1}, s_{2}$ & 1 & $\epsilon^{2}$ & $\frac{\epsilon^{\prime 2}}{\epsilon} \beta$ & $\epsilon$ \\
$\gamma \leftrightarrow s_{1}, s_{2}$ & $\epsilon^{2}$ & 1 & $\epsilon \epsilon^{\prime} \beta$ & $\epsilon$ \\
\hline \hline
\end{tabular}

TABLE II. Oscillation amplitudes in the case of rank-1 